\title{
Metronidazole resistance: a hidden epidemic?
}

\author{
A. Smith
}

\section{Key points}

Discusses the front line of clinical microbiology, reflecting on the battle against antimicrobial resistance.

\begin{tabular}{l|l}
$\begin{array}{l}\text { Reflects on why clinical microbiologists } \\
\text { might be missing the extent of }\end{array}$ & $\begin{array}{l}\text { Highlights the role of a dental team } \\
\text { approach to antimicrobial stewardship } \\
\text { metronidazole resistance. }\end{array}$ \\
in dental practice.
\end{tabular}

Alerts dental leaders and the general public of the diminishing access to expert advice by the dental team with the demise of clinical oral microbiologist posts.

This is a personal reflection from working as a clinical microbiologist and becoming increasingly concerned over gaps in our knowledge of metronidazole resistance and its impact on clinical outcome.

World antibiotic awareness week is a campaign led by the World Health Organisation and was run between 13-19 November last year. The aim of the campaign is to raise awareness of the threat of antibiotic resistance to clinical care. Within the UK the Association of Clinical Oral Microbiologists (ACOM), working in partnership with a number of organisations including the Faculty of General Dental Practitioners, utilises an annual 'Thunderclap' campaign to raise the awareness of appropriate antimicrobial use in dentistry. During the last 18 months I have been made increasingly aware of the emergence of bacterial resistance to metronidazole, a barrier I thought was too costly (in terms of bacterial metabolism) for anaerobes to cross and result in clinically relevant infections. I should have known better.

Metronidazole is a member of the nitroimidazole group of compounds with antimicrobial and anti-protozoan activity (such as Trichomonas vaginalis) developed in the late 1950s. Its link to the management of dental infections was first reported in 1962 as a coincidental finding of the 'double' resolution of trichomonal vaginitis and 'acute marginal gingivitis' and the rest as they say is history. ${ }^{1}$. In common with other anti-microbial agents,

${ }^{1}$ College of Medical, Veterinary \& Life Sciences, Glasgow Dental Hospital \& School, University of Glasgow, 378

Sauchiehall Street, Glasgow, G2 3JZ

Correspondence to: Andrew Smith

Email: andrew.smith@glasgow.ac.uk

Refereed Paper. Accepted 5 January 2018

DOI: $10.1038 /$ sj.bdj.2018.221 resistance to metronidazole can take many forms especially under selection pressure according to basic Darwinian principles when levels of the antimicrobial in the environment rise. The main driver for the development of antimicrobial resistance is exposure to antibiotics - high levels of antibiotic use are correlated with high levels of resistance. ${ }^{2}$ But first let's look at some clinical evidence. I first became alerted to the clinical implications of metronidazole resistance when in a relatively short time, we became aware in Glasgow of at least two clinically significant infections caused by metronidazole resistant bacteria. One of these was a knee joint infection with an anaerobic streptococci (also found in periodontal disease and dental abscesses) and the other was a Bacteroides thetaiotaomicron blood stream infection. These isolates are reported in last year's report of antimicrobial resistance in Scotland ${ }^{3}$ where metronidazole resistance was included in annual surveillance data for the first time. Looking more widely, other workers have noted the appearance of metronidazole resistance in microbial species that can also be recovered from dental infections. ${ }^{4,5}$ The logical extension of this data is that it will only be a matter of time before metronidazole resistance in oral infections is reported. Metronidazole resistance can occur by a number of different mechanisms that involve reduced uptake of the drug, increased removal from the bacterial cell or by reducing the rate of metronidazole activation inside anaerobes. Of immediate concern is the occurrence of specific resistance genes (nim) which code for an alternative set of enzymes that can convert activated forms of metronidazole into non-toxic derivatives. ${ }^{6} \mathrm{~A}$ family of nim genes is known to exist and can spread between different classes of bacteria.

It is difficult to define the extent of the problem of metronidazole resistance; firstly, there is the paradox of detecting metronidazole resistance in the laboratory. Screening of primary agar plates from infection specimens for the presence of anaerobes is usually performed by assessing zones of inhibition caused by a metronidazole disc. Following anaerobic growth, any microbe that is not inhibited by metronidazole is discarded since a commonly used definition of an anaerobe is an isolate that is inhibited by metronidazole! In clinical dental practice a further challenge to understanding the extent of the problem is the collection, submission, reporting and surveillance of dento-alveolar infections. There is no quick and easy fix for this, there are multiple challenges and we have tried (and failed) in Glasgow to facilitate this. ${ }^{7}$ However, this does not mean the issue should not be re-examined and utilise new technologies. We do know that increased levels of prescribing lead to increased patterns of resistance and perhaps an easier fix than obtaining surveillance data is to review dental prescribing of metronidazole.

Current prescribing data by GDPs in England and Scotland demonstrates that approximately $60 \%$ and $52 \%$, respectively, of the total metronidazole prescribed in primary care is for dental infections. ${ }^{8,9}$ It is difficult to understand why metronidazole is so widely prescribed in dental practice because the first 
line of treatment for acute dental infections should be infection source control, that is, surgical treatment (extraction, endodontics, incision and drainage, debridement etc). If required then the first line antimicrobial agent is a beta-lactam (penicillin, amoxicillin). ${ }^{10}$ The dental team should become more aware of the importance of antimicrobial stewardship to conserve metronidazole.

There is also an important role here for the current senior leaders in dentistry; antimicrobial stewardship is a team process with clinical oral microbiologists playing a vital role within the team. Clinical oral microbiologists have the training, dental background, expertise and competencies to facilitate antimicrobial stewardship, yet there are only eight on the GDC Specialist Register (and not all of these are in clinical practice). ACOM believes there is overwhelming evidence now that all dental hospitals and schools should have access to a consultant clinical oral microbiologist, not just for stewardship expertise but also their expertise in infection prevention and control. These two subjects are not mutually exclusive.

I just hope that enough clinical oral microbiologists can be trained in time for the next generation of the dental team before we completely lose metronidazole from the drug cabinet.

1. Shinn D L S. Metronidazole in acute ulcerative gingivitis. Lancet 1962; 279: 1191.

2. Goossens $\mathrm{H}$, Ferech M, Vander S R, Elseviers M. Outpatient antibiotic use in Europe and association with resistance: a cross-national database study. Lancet 2005; 365: 579-587.

3. Health Protection Scotland and Information Services Division. Scottish antimicrobial use and resistance in humans in 2015. 2016. Available at http://www.isdscotland.org/Health-Topics/Prescribing-and-Medicines/ Publications/2016-08-30/2016-08-30-SAPG-2015Report.pdf (accessed November 2017).
4. Veloo A C, Boiten K E, Wekema-Mulder G J. Antibiotic susceptibility profiles of Prevotella species in the Netherlands. Int J Antimicrob Agents 2015; 45: 554-556.

5. Veloo A C, Welling G W, Degener J E. Antimicrobial susceptibility of clinically relevant Gram-positive anaerobic cocci collected over a three-year period in the Netherlands. Antimicrob Agents Chemother 2011; 55: 1199-1203.

6. Leiros H K, Kozielski-Stuhrmann S, Kapp U, Terradot L, Leonard G A, McSweeney S M. Structural basis of 5-nitroimidazole antibiotic resistance: the crystal structure of NimA from Deinococcus radiodurans. J Biol Chem 2004; 279: 55840-55849.

7. Roy K, Smith A J, Sanderson J et al. Barriers to the use of a diagnostic oral microbiology laboratory by general dental practitioners. Br Dent J 1999 186: 345-347.

8. Health and Social Care Information Centre. Prescription cost analysis - dental data 2015. 2016. Available at https://digital.nhs.uk/catalogue/PUB20200 (accessed November 2017).

9. Health Protection Scotland. Scottish One Health Antimicrobial use and Antimicrobial Resistance Report 2016. 2017. Available at http://www.hps.scot.nhs.uk/pubs/ detail.aspx?id=3378\# (accessed November 2017).

10. Faculty of General Dental Practitioners. Antimicrobial prescribing for GDPs. Available at https://www.fgdp.org uk/publication/antimicrobial-prescribing-gdps (accessed November 2017). 